\title{
Lipid Binding Regulates Synaptic Targeting of PICK1, AMPA Receptor Trafficking, and Synaptic Plasticity
}

\author{
Wenying Jin, ${ }^{1}$ Woo-Ping Ge, ${ }^{2}$ Junyu Xu, ${ }^{1}$ Mian Cao, ${ }^{1}$ Lisheng Peng, ${ }^{1}$ Wingho Yung, ${ }^{3}$ Dezhi Liao, ${ }^{4}$ Shumin Duan, ${ }^{2}$ \\ Mingjie Zhang, ${ }^{1}$ and Jun $\mathrm{Xia}^{1}$ \\ ${ }^{1}$ Department of Biochemistry, The Hong Kong University of Science and Technology, Clear Water Bay, Kowloon, Hong Kong, China, ${ }^{2}$ Institute of \\ Neuroscience, Shanghai Institutes of Biological Sciences, Chinese Academy of Sciences, Shanghai 200031, China, ${ }^{3}$ Department of Physiology, Faculty of \\ Medicine, The Chinese University of Hong Kong, Shatin, Hong Kong, China, and ${ }^{4}$ Department of Neuroscience, University of Minnesota, Minneapolis, \\ Minnesota 55455
}

The targeting and surface expression of membrane proteins are critical to their functions. In neurons, synaptic targeting and surface expression of AMPA-type glutamate receptors were found to be critical for synaptic plasticity such as long-term potentiation and long-term depression (LTD). PICK1 (protein interacting with C kinase 1) is a cytosolic protein that interacts with many membrane proteins, including AMPA receptors via its PDZ (postsynaptic density-95/Discs large/zona occludens-1) domain. Its interactions with membrane proteins regulate their subcellular targeting and surface expression. However, the mechanism by which PICK1 regulates protein trafficking has not been fully elucidated. Here, we show that PICK1 directly binds to lipids, mainly phosphoinositides, via its BAR (Bin/amphiphysin/Rvs) domain. Lipid binding of the PICK1 BAR domain is positively regulated by its PDZ domain and negatively regulated by its $\mathrm{C}$-terminal acidic domain. Mutation of critical residues of the PICK1 BAR domain eliminates its lipid-binding capability. Lipid binding of PICK1 controls the subcellular localization of the protein, because BAR domain mutant of PICK1 has diminished synaptic targeting compared with wild-type PICK1. In addition, the BAR domain mutant of PICK1 does not cluster AMPA receptors. Moreover, wild-type PICK1 enhances synaptic targeting of AMPA receptors, whereas the BAR domain mutant of PICK1 fails to do so. The BAR domain mutant of PICK1 loses its ability to regulate surface expression of the AMPA receptors and impairs expression of LTD in hippocampal neurons. Together, our findings indicate that the lipid binding of the PICK1 BAR domain is important for its synaptic targeting, AMPA receptor trafficking, and synaptic plasticity.

Key words: PICK1; AMPA receptor; synaptic plasticity; LTD; PDZ domain; BAR domain

\section{Introduction}

Regulated insertion and internalization of AMPA-type glutamate receptors at synapses are important molecular mechanisms of synaptic plasticity, including long-term potentiation (LTP) and long-term depression (LTD) (Bredt and Nicoll, 2003; Park et al., 2004). Several lines of evidence indicate that the $C$ termini of AMPA receptors are crucial for their synaptic targeting and surface expression (Malinow and Malenka, 2002). A number of intracellular proteins that interact with the $\mathrm{C}$ termini of AMPA receptors have been identified (Barry and Ziff, 2002; Song and Huganir, 2002; Henley, 2003). One such protein, PICK1 (protein interacting with $\mathrm{C}$ kinase 1 ), is a cytosolic protein containing an $\mathrm{N}$-terminal PDZ [postsynaptic density-95 (PSD-95)/Discs large/ zona occludens-1] domain, a central BAR (Bin/amphiphysin/

Received June 12, 2005; revised Jan. 10, 2006; accepted Jan. 11, 2006.

This work was supported in part by grants from the Research Grants Council of the Hong Kong Special Administrative Region, China (HKUST6144/03M and HKUST6130/04M). We thank W. Tung and C. He for their expert technical assistance; C. Wong and Dr. Z. Wu for help with the fluorescence microscopy; Drs. R. Huganir and D. Banfield for helpful discussions; and Dr. N. Ip, Dr. M. Sheng, and Naikei Wong for critical reading of this manuscript.

Correspondence should be addressed to Dr. Jun Xia, Department of Biochemistry, The Hong Kong University of Science and Technology, Clear Water Bay, Kowloon, Hong Kong, China. E-mail: jxia@ust.hk.

DOI:10.1523/JNEUROSCI.3503-05.2006

Copyright $\odot 2006$ Society for Neuroscience $\quad$ 0270-6474/06/262380-11\$15.00/0
Rvs) domain, and an acidic domain close to the $\mathrm{C}$ terminus (supplemental Fig. S1 $A$, available at www.jneurosci.org as supplemental material). The PDZ domain of PICK1 interacts with the C termini of AMPA receptor subunits (Dev et al., 1999; Xia et al., 1999). PICK1 colocalizes with the AMPA receptors at excitatory synapses and clusters the AMPA receptors when coexpressed in heterologous cells. The interaction of PICK1 with AMPA receptors was found to regulate surface expression of the AMPA receptors in a protein kinase-dependent manner (Matsuda et al., 1999; Chung et al., 2000; Perez et al., 2001; Terashima et al., 2004). Furthermore, the binding of PICK1 to AMPA receptors was found to be critical to AMPA receptor trafficking during synaptic plasticity (Matsuda et al., 2000; Xia et al., 2000; Kim et al., 2001). In a more recent study, PICK1 knock-out mice were found to be deficient in the activity-dependent insertion of glutamate receptor 2 (GluR2)-containing AMPA receptors (Gardner et al., 2005).

In addition to its PDZ domain, PICK1 was previously predicted to have a coiled-coil domain in the middle of the protein (Xia et al., 1999). Recently, a crystallographic study of the amphiphysin BAR domain suggested that this predicted coiled-coil domain is likely part of a larger BAR domain (Peter et al., 2004). The BAR domain is found in many proteins that are involved in 
protein trafficking, especially endocytosis. The BAR domain of amphiphysin can directly bind and tubulate liposomes (Takei et al., 1999; Peter et al., 2004). In addition, amphiphysin was also found to interact with adaptor protein 2 (AP2), clathrin, dynamin, and synaptojanin and to be critically involved in the endocytosis of synaptic vesicles (Wigge and McMahon, 1998; Slepnev and De Camilli, 2000). Surprisingly, the structure of the amphiphysin BAR domain was found to be highly similar to the structure of arfaptin2 (Tarricone et al., 2001), a protein that shares high sequence homology with the central region of PICK1 (supplemental Fig. S1 B, available at www.jneurosci.org as supplemental material). By virtue of this structural and amino acid sequence similarity, PICK1 is predicted to possess a BAR domain. The coexistence of a BAR domain and a PDZ domain in PICK1 raises the interesting possibility that PICK1 could couple membrane proteins to the protein trafficking machinery. Here, we show that PICK1 can indeed directly bind to lipids via its BAR domain. This lipid binding capability is required for the synaptic targeting of PICK1, trafficking of AMPA receptors, and synaptic plasticity.

\section{Materials and Methods}

cDNA cloning, mutagenesis, and protein purification. Rat PICK1 cDNA (Xia et al., 1999) constructs were subcloned into corresponding expression vectors in frames by restriction enzyme digestion and ligation. To generate BAR domain mutants of PICK1, we synthesized PCR primers containing the desired mutation. PCR mutagenesis was performed using a Quikchange site-directed mutagenesis kit (Stratagene, La Jolla, CA). All mutations were subsequently confirmed by sequencing. To produce fusion proteins, cDNA constructs were transformed into Escherichia coli BL21 cells and induced with isopropyl- $\beta$-D-thiogalactopyranoside. Glutathione $S$-transferase (GST) fusion proteins were affinity purified by glutathione Sepharose-4B (Amersham Biosciences, Uppsala, Sweden), and maltose-binding protein (MBP) fusion protein was affinity purified by amylose resin (New England Biolabs, Beverley, MA), according to the instructions of the manufacturer. Purified fusion proteins were eluted and dialyzed against the corresponding buffers for follow-up experiments. Fusion protein concentrations were determined by Coomassie assays (Pierce, Rockford, IL).

Lipid binding assay. The lipid binding assay was performed by following the procedure described by Peter et al. (2004). Specifically, brain lipid extracts (Folch fraction I, B1502; Sigma, St. Louis, MO) were resuspended at $2 \mathrm{mg} / \mathrm{ml}$ in a buffer containing $20 \mathrm{~mm}$ HEPES, pH 7.4, $150 \mathrm{~mm}$ $\mathrm{NaCl}$, and $1 \mathrm{~mm}$ DTT. The fusion protein $(5 \mu \mathrm{M})$ was incubated with 0.6 $\mathrm{mg} / \mathrm{ml}$ liposomes in $100 \mu \mathrm{l}$ of buffer for $15 \mathrm{~min}$ at $37^{\circ} \mathrm{C}$ and then spun at $140,000 \times g$ for $15 \mathrm{~min}$ at $4^{\circ} \mathrm{C}$ in a Beckman TLA100.1 rotor (Beckman Instruments, Fullerton, CA). After removing all supernatants, the pellets were washed once with the same buffer solution and brought to the same volume as that of the supernatant. The supernatant and the pellet proteins were subjected to SDS-PAGE and visualized by Coomassie stain assays.

Lipid strip assay. The phosphatidylinositol phosphate (PIP) strips (P6001; Echelon Biosciences, Salt Lake City, UT) were blocked in 3\% fatty acid-free BSA in TBS-T (10 mm Tris, pH 8.0, $150 \mathrm{~mm} \mathrm{NaCl}, 0.1 \%$ Tween 20) at room temperature for $1 \mathrm{~h}$. The strips were then incubated with 1 $\mu \mathrm{g} / \mathrm{ml}$ MBP-PICK1 fusion proteins at room temperature for $2 \mathrm{~h}$. After incubation with proteins, the strips were washed five times in TBS-T and then incubated for $1 \mathrm{~h}$ with the anti-PICK1 C-100 antibody (Xia et al., 1999) at room temperature. After washing, the strips were incubated with horseradish peroxidase-conjugated anti-rabbit antibody (1:5000; Amersham Biosciences, Buckinghamshire, UK) for $1 \mathrm{~h}$ at room temperature, followed by washing in TBS-T. The signals were detected by enhanced chemiluminescence (PerkinElmer Life Sciences, Boston, MA).

Human embryonic kidney 293 T cell culture, transfection, and immunostaining. Human embryonic kidney 293T (HEK293T) cells were cultured in MEM media (Invitrogen, Grand Island, NY) plus fetal bovine serum. For immunostaining, HEK293T cells were grown on coverslips coated with $0.2 \%$ gelatin. cDNA constructs of GluR2, green fluorescent protein (GFP)-tagged wild-type PICK1, or mutant PICK1 were transfected into the HEK293T cells by calcium phosphate coprecipitation. The cells were fixed $36-48 \mathrm{~h}$ after transfection by $4 \%$ paraformaldehyde and $4 \%$ sucrose in PBS for $20 \mathrm{~min}$ at room temperature. The cells were then permeabilized by $0.2 \%$ Triton X-100 in PBS for $10 \mathrm{~min}$ at room temperature. After blocking with $10 \%$ normal donkey serum (NDS) in PBS for $1 \mathrm{~h}$, the cells were incubated with affinity-purified rabbit anti-GluR2/3 antibody in $3 \%$ NDS for $1 \mathrm{~h}$ at room temperature, followed by $1 \mathrm{~h}$ of incubation with Red-X-conjugated fluorescence anti-rabbit secondary antibody (Jackson ImmunoResearch, West Grove, PA). After washing with PBS, the coverslips were mounted with Permafluor (Immunon, Pittsburgh, PA). The cells were observed with a Nikon Eclipse TE2000 (Nikon, Tokyo, Japan) inverted fluorescence microscope under a $60 \times$ or a $100 \times$ Plan Apochromatic oil lens [numerical aperture (NA), 1.4; Nikon). Pictures were taken by a monochrome low noise cooled CCD camera (SPOT-RT; Diagnostic Instruments, Sterling Heights, MI) controlled by MetaMorph imaging acquisition software (Universal Imaging, West Chester, PA). Images were processed with Adobe Photoshop (Adobe Systems, San Jose, CA) to adjust intensity and contrast, to select the region of interest, and to overlay two images. All images were taken in monochrome gray scale and artificially colored for presentation.

Yeast two-hybrid assay. To test the interaction between GluR2 and PICK1 or PICK1 self-association, the corresponding DNA constructs were subcloned in frames into pPC86 vectors that contained the GAL4 activation domain or PPC97 vectors that contained the GAL4 DNA binding domain (Xia et al., 1999). The constructs were cotransformed into HF7c yeast cells and grown on double-minus (lacking leucine, tryptophan) plates. Positive clones were selected and tested on triple-minus plates (lacking leucine, tryptophan, and histidine). After the growth selection, the clones were further tested for $\beta$-galactosidase activity.

Coimmunoprecipitation. HEK293T cells were cotransfected with glutamate receptor-interacting protein 1 (GRIP1) and mycPICK1 or mycPICK1 2K-E. Two days after transfection, the HEK293T cells were lysed by $2 \%$ Triton X-100 in PBS and incubated with antibody/protein A complex at $4^{\circ} \mathrm{C}$ for at least $2 \mathrm{~h}$. The resin was washed once with cold PBS and $1 \%$ Triton X-100, twice with cold PBS, $1 \%$ Triton X-100, and $500 \mathrm{~mm}$ $\mathrm{NaCl}$, and three times with cold PBS. After washing, the resin was eluted with $1 \times$ SDS sample buffer and was analyzed by SDS-PAGE and immunoblotted with affinity-purified rabbit anti-GRIP1 or PICK1 antibody.

Generation of Sindbis viruses. The cDNA encoding GFP-tagged wildtype and mutant PICK1 proteins were subcloned into the pSinRep5 viral vector (Invitrogen, Carlsbad, CA). The pSinRep5 constructs and DH26S helper DNA (Invitrogen) were then subjected to restriction enzyme linearization, DNA purification, and in vitro transcription using an SP6 promotor in vitro transcription kit (Ambion, Austin, TX). For production of viruses, mRNAs were transfected into baby hamster kidney cells by Lipofectamine 2000 (Invitrogen). Culturing media were removed $36-48 \mathrm{~h}$ after transfection and centrifuged at $2000 \mathrm{~g}$ for $10 \mathrm{~min}$ at $4^{\circ} \mathrm{C}$ to remove all cell debris. The supernatants were further centrifuged at $20,000 \times g$ at $4^{\circ} \mathrm{C}$ for $4 \mathrm{~h}$ to harvest the virus particles. The viruses were aliquoted and stored at $-80^{\circ} \mathrm{C}$. To infect the neurons, the viruses were directly added to the culture media at a titration determined for each batch of viruses. One day after infection, the neurons were fixed and stained for imaging analysis.

Neuronal culture, staining, and quantification. Cultured hippocampal or cortical neurons were prepared from embryonic day 18 Sprague Dawley rats and grown on coverslips coated with poly-L-lysine (Sigma). The hippocampal neurons were infected with Sindbis viruses between 16 and $24 \mathrm{~d}$, and the cortical neurons were transfected by calcium phosphate coprecipitation between 13 and $15 \mathrm{~d}$ in vitro. For staining, neurons were fixed by $4 \%$ paraformaldehyde plus $4 \%$ sucrose in PBS for $15 \mathrm{~min}$ at $4^{\circ} \mathrm{C}$. The neurons were further treated with $-20^{\circ} \mathrm{C}$ methanol for $10 \mathrm{~min}$, washed three times with $0.03 \%$ Triton X-100, and then permeabilized by $0.2 \%$ Triton X-100 for $10 \mathrm{~min}$ at $4^{\circ} \mathrm{C}$. After blocking with $10 \%$ normal donkey serum for $>2 \mathrm{~h}$ at room temperature, the neurons were incubated with primary antibody in $3 \% \mathrm{NDS}$ at $4^{\circ} \mathrm{C}$ overnight to stain their endogenous proteins or $1 \mathrm{~h}$ at room temperature for overexpressed protein staining. For endogenous GluR1 and GluR2 staining, antibodies 
against the C termini of GluR1 and GluR2 were used, and the GluR2 antibody was directly coupled to Cy3 (Liao et al., 1999). GFP-GluR2transfected neurons were first incubated with GFP antibody in a medium at $10^{\circ} \mathrm{C}$ for $30 \mathrm{~min}$ to stain the surface GluR2 before fixation. Fluorescent secondary antibodies were added at room temperature for $1 \mathrm{~h}$. After washing, coverslips were mounted and observed under a fluorescence microscope. Quantitative immunofluorescence was performed under a $60 \times$ Plan Apochromatic oil lens (1.4 NA; Nikon Instech). Data were acquired and quantified using MetaMorph acquisition and analysis software (Universal Imaging, West Chester, PA). Synaptic clusters were determined by a threshold set at twice the average dendritic gray value. To obtain the surface-to-total GFP-GluR2 ratio, the surface and total gray values of individual clusters were first normalized to dendritic gray to obtain the relative gray value of each individual cluster. The surface-tototal GFP-GluR2 ratio was then obtained by dividing the relative surface gray value of each cluster by its corresponding relative total gray value.

Biotinylation assay. HEK293T or COS7 cells were washed three times with PBS supplemented with $0.5 \mathrm{~mm} \mathrm{CaCl}_{2}$ and $0.5 \mathrm{~mm} \mathrm{MgCl}_{2}$ (B buffer) and treated with $0.5 \mathrm{mg} / \mathrm{ml}$ sulfo-succinimidyl-6-(biotinamido) hexanoate (sulfo-NHS-LC-biotin; Pierce) in B buffer for $5 \mathrm{~min}$ at room temperature or $30 \mathrm{~min}$ at $4^{\circ} \mathrm{C}$. The free sulfo-NHS-LC-biotin was removed by rapidly washing the cells two times with $100 \mathrm{~mm}$ glycine in $\mathrm{B}$ buffer followed by two washes with B buffer. The biotinylated cells were solubilized with $1 \mathrm{ml}$ of radioimmunoprecipitation assay buffer $(10 \mathrm{~mm}$ Tris, pH 7.4, 150 mм NaCl, 1 mм EDTA, 0.1\% SDS, 1\% Triton X-100, 1\% sodium deoxycholate). The samples were centrifuged at maximum speed in a table centrifuge for $15 \mathrm{~min}$ at $4^{\circ} \mathrm{C}$. A sample of this supernatant was removed for estimation of the total protein. The remaining supernatants were incubated with $100 \mu \mathrm{l}$ of $50 \%$ slurry of neutravidin beads (Pierce) for $1 \mathrm{~h}$ at $4^{\circ} \mathrm{C}$ with constant rotation. After several washes, the biotinylated surface proteins were eluted from the neutravidin beads in $100 \mu \mathrm{l}$ of $1 \times$ SDS sample buffer. The samples were subjected to SDS-PAGE and Western blot analysis. Instead of using ECL and x-ray film for the Western blot analysis and quantification, we used an Opti-4CN Substrate kit (Bio-Rad, Hercules, CA) to detect protein expression on the polyvinylidene difluoride (PVDF) membrane. The PVDF membrane was incubated in a solution containing $2 \%$ Opti-4CN substrate for $15 \mathrm{~min}$ and washed several times. In our tests, this method provided better linearity for densitometry analysis compared with the ECL and x-ray film exposure method. The surface GluR2 ratio was calculated only when a linear standard curve was obtained.

Electrophysiology. Hippocampal slices were prepared as described previously (Yang et al., 2003). The use and care of animals in this study followed the guidelines of the Shanghai Institutes for Biological Sciences Animal Research Advisory Committee. In brief, postnatal rats (14-17 d of age) were anesthetized with sodium pentobarbital. After decapitation, hippocampal formations were dissected rapidly and placed in an ice-cold oxygenated $\left(95 \% \mathrm{O}_{2}\right.$ and $\left.5 \% \mathrm{CO}_{2}\right)$ solution containing the following (in mM): $119 \mathrm{NaCl}, 2.5 \mathrm{KCl}, 2.5 \mathrm{CaCl}_{2}, 1.3 \mathrm{MgSO}_{4}, 1 \mathrm{NaH}_{2} \mathrm{PO}_{4}, 26.2$ $\mathrm{NaHCO}_{3}$, and 11 glucose, $\mathrm{pH}$ 7.3. Transverse slices (400 $\mu \mathrm{m}$ thick) were cut with a vibratome (Campden Instruments, Loughborough, UK) and maintained in an incubation chamber for $\geq 2 \mathrm{~h}$ at $25^{\circ} \mathrm{C}$ before recording. During experiments, individual slices were transferred to a submersionrecording chamber and were continuously perfused with the aboveoxygenated solution $(3.0 \mathrm{ml} / \mathrm{min})$ at $28^{\circ} \mathrm{C}$. Slices were visualized with infrared optics using an Olympus microscope (BX50WI; Olympus Optical, Hamburg, Germany) equipped with DIC optics.

Whole-cell patch recordings were made from CA1 pyramidal neurons in the presence of $\mathrm{GABA}_{\mathrm{A}}$ antagonist bicuculline $(10 \mu \mathrm{M})$. Recording pipettes were filled with a solution containing the following (in $\mathrm{mM}$ ): 125 Cs-gluconate, $5 \mathrm{CsCl}, 10 \mathrm{HEPES}, 8 \mathrm{NaCl}, 3 \mathrm{Na}_{2} \mathrm{ATP}, 0.3 \mathrm{Na}$-GTP, 0.2 EGTA, $10 \mathrm{Na}_{2}$-phosphocreatine, 10 tetraethylammonium-Cl, $5 \mathrm{~N}$-ethyl bromide quaternary salt, $\mathrm{pH}$ 7.3. The membrane potential was maintained at -65 to $-70 \mathrm{mV}$. Constant current pulses $(10-20 \mu \mathrm{A}, 100 \mu \mathrm{S}$, $0.0167 \mathrm{~Hz})$ were applied through extracellular stimulating electrodes (MCE-100; Rhodes Medical Instruments, Woodland Hills, CA) placed at Schaffer collateral of the CA1 region to induce EPSCs in the pyramidal neurons. MBP, wild-type PICK1, and PICK1 2K-E fusion proteins were added into the intracellular solution at a final concentration of $50 \mu \mathrm{g} / \mathrm{ml}$.

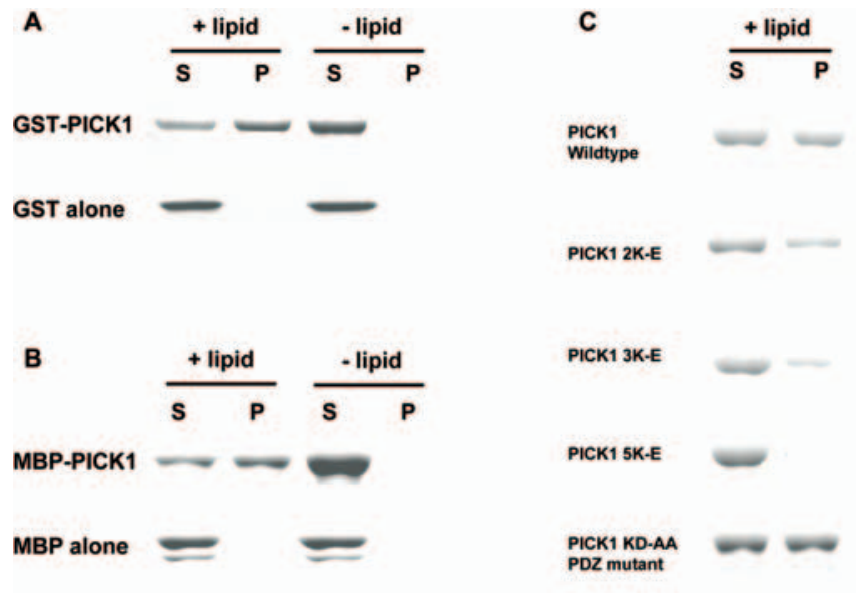

Figure 1. The BAR domain of PICK1 binds to lipid. $\boldsymbol{A}$, GST-PICK1s were mixed with brain lipid extract and subjected to high-speed centrifugation to spin down the liposomes. Pellets were redissolved in the same buffer and brought to the same volume as the supernatant. Equal amounts of pellet and supernatant were loaded and resolved by SDS-PAGE. The proteins were then stained by Coomassie blue. A substantial amount of PICK1 was found to accompany the liposome and show up in the pellet (top, left two lanes; $S$, supernatant; $P$, pellet.). Without lipid, PICK1 was not found in the pellet (top, right two lanes). As a control, GST itself was incubated with liposome and subjected to the same centrifugation. No GST was found in the liposomecontaining pellet (bottom). $\boldsymbol{B}$, Similar experiments were performed as in $\boldsymbol{A}$ using MBP-PICK1s. Similar to GST-PICK1, MBP-PICK1 was found in the liposome-containing fraction (top line), but MBP alone was not (bottom line). $\boldsymbol{C}$, The lipid binding capability of the BAR domain mutations and the PDZ domain mutant were tested. BAR domain mutants $2 K-E, 3 K-E$, and $5 K-E$ all significantly reduced the binding of PICK1 with lipids, whereas PDZ mutant K27D28-AA did not.

In the experiments to test LTD, data were collected for at least $10 \mathrm{~min}$ after breaking-in to allow a full exchange between the cytoplasm and the pipette solutions. LTD was induced with 300 stimuli $(1 \mathrm{~Hz})$ at $-40 \mathrm{mV}$ (Dudek and Bear, 1992; Mulkey and Malenka, 1992; Daw et al., 2000), with the same intensity as the test stimulus for evoking EPSCs. Data were accepted for analysis only when the postsynaptic currents or voltages and the input resistance did not vary $>15 \%$ of the average values during the control period. Signals filtered at $5 \mathrm{kHz}$ using the amplifier circuitry were sampled at $10 \mathrm{kHz}$ and analyzed using Clampex 8.2 (Molecular Devices, Union City, CA). Data were presented as means \pm SEM, and statistical differences were determined by the Student's $t$ test. Reagents were obtained from Sigma-Aldrich except where noted.

\section{Results}

\section{The BAR domain of PICK1 binds to lipids}

Because PICK1 is predicted to contain a BAR domain, we first set out to test whether PICK1 binds to lipids. GST-tagged PICK1 fusion protein (ST-PICK1) was incubated with liposomes and then subjected to high-speed centrifugation. Proteins that bind to lipids will be spun down together with liposomes and found in the pellet. We found that GST-PICK1 readily associates with liposomes, because substantial amounts of GST-PICK1 were found in the liposome-containing pellet (Fig. $1 \mathrm{~A}$ ). In contrast, GST-PICK1 was not found in the pellet when the liposomes were omitted from the reaction mixture. As a control, GST alone was not found in pellets with or without liposomes. To rule out the possibility that the GST tag may cause some undesirable conformational changes to PICK1 and lead to its lipid binding, we generated MBP-tagged PICK1 fusion proteins (MBP-PICK1) and repeated the lipid binding experiments. As shown in Figure $1 B$, similar to GST fusion proteins, MBP-PICK1 was found to bind to lipids, whereas MBP alone was not.

We then determined the residues of PICK1 that are critical to its lipid binding. The amphiphysin BAR domain forms a 
A

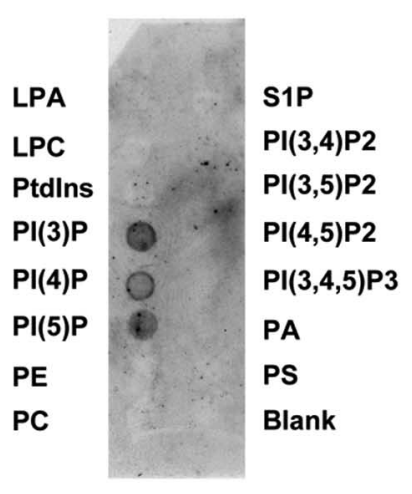

B

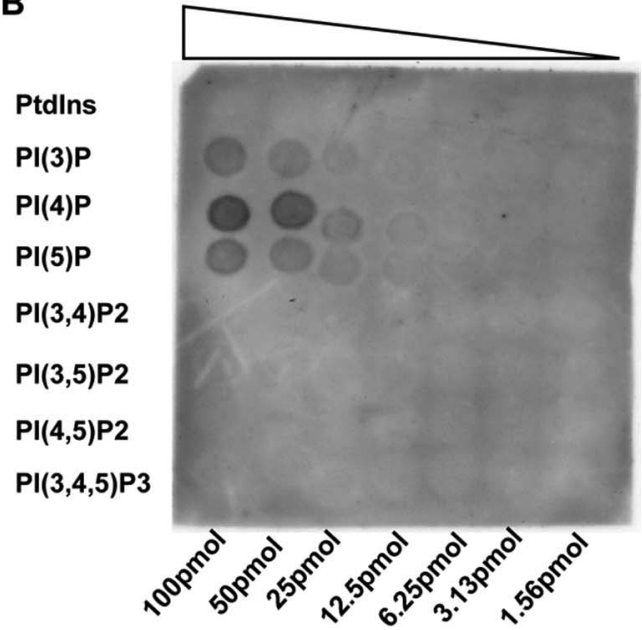

Figure 2. PICK1 preferably binds to phosphoinositides. MBP-PICK1 fusion proteins were overlaid onto a nitrocellulose membrane spotted with 15 different kinds of lipid molecules (100 pmol each) $(\boldsymbol{A})$ or different concentrations of lipids $(\boldsymbol{B})$. Proteins bound to lipids were detected with the anti-PICK1 antibody. PE, Phosphatidylethanolamine; PC, phosphatidylcholine; PS, phosphatidylserine; PA, phosphatic acid; LPA, lysophosphatidic acid; LPC, lysophosphacholine; S1P, sphingosine-1-phosphate.

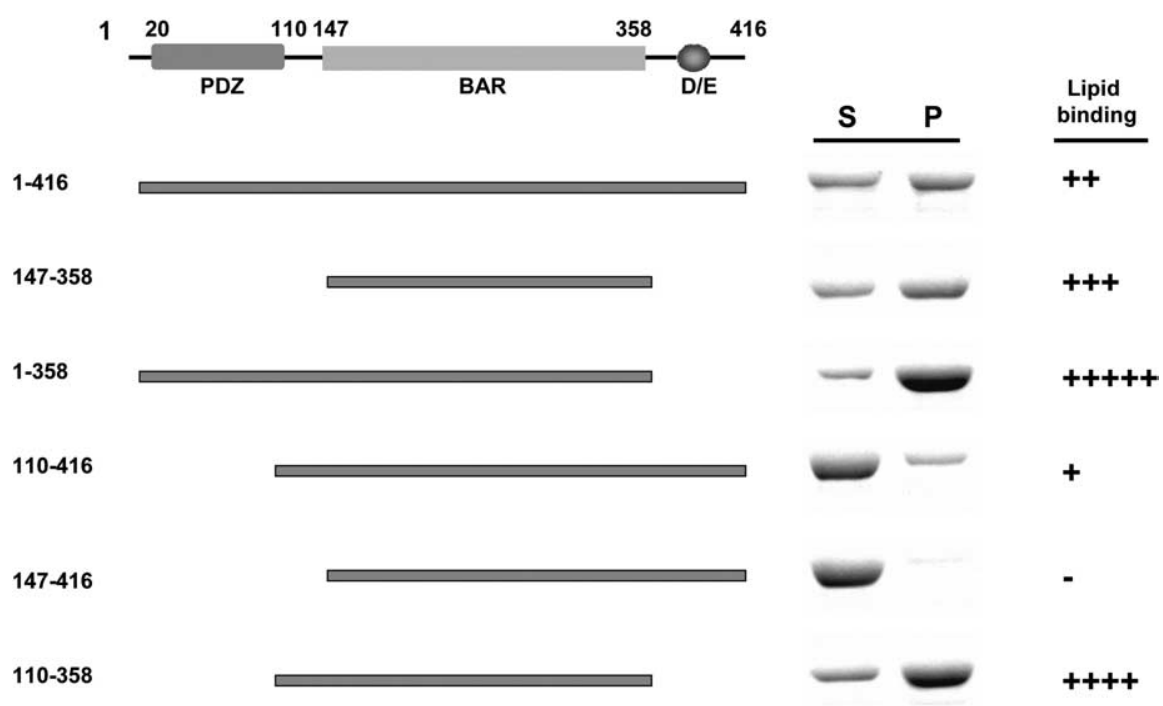

Figure 3. The intramolecular regulation of lipid binding of PICK1. Truncation mutants of PICK1 were tested for their lipid binding capabilities. The lipid binding capability of the BAR domain of PICK1 is negatively regulated by the acidic domain and positively regulated by the PDZ domain and the linker region between the PDZ and BAR domain of PICK1. The domain structure of PICK1 is shown on the top. D/E indicates the acidic domain with nine aspartic acids or glutamic acids. The length of each fusion protein is represented by a solid line, with the first and the last amino acid residues indicated on the left. The lipid binding result of each fusion protein is shown on the right. S, Supernatant; $P$, pellet.

mutation of lysines 266 and 268 to glutamate eliminated the majority of the lipid binding capability of PICK1. The region of PICK1 corresponding to the second pair of positively charged residues of the amphiphysin BAR domain is more divergent. There are three lysines (K251, 252, 257) that are likely to be responsible for lipid binding (supplemental Fig. $\mathrm{S} 1 B$, available at www.jneurosci.org as supplemental material). We substituted glutamate for lysines 251, 252, and 257 and tested the lipid binding ability of the mutant (PICK1 3K-E). As shown in Figure $1 C$, substitution of glutamate for lysines 251, 252, and 257 also significantly reduced the lipid binding capability of PICK1. We also mutated all five lysines (PICK1 5K-E). As expected, this pentalysine mutant of PICK1 had minimal lipid binding capability. As a control, we also tested the lipid binding capability of the PICK1 PDZ mutant, in which two critical residues (K27 and D28) of the PDZ domain were changed to alanine. The PICK1 PDZ mutant was found to have similar lipid binding capability to that of wild-type PICK1.

To determine what kinds of lipids interact with PICK1, we performed a lipid strip assay. Purified PICK1 fusion protein was incubated with a lipid strip containing 15 different types of lipid molecules spotted on a nitrocellulose membrane. After washing, PICK1 was detected by immunoblotting. We found that PICK1 preferably binds to phosphoinositides (PtdIns) (Fig. $2 A)$. Specifically, PICK1 binds strongly to PtdIns(3)P, PtdIns(4)P, and PtdIns(5)P. This result was confirmed by probing PIP arrays, which contain a different concentration of phosphoinositides spotted on nitrocellulose membranes. The results from the PIP arrays showed that PICK1 also binds to PtdIns(3)P, PtdIns(4)P, and PtdIns(5)P with relatively high affinity (Fig. 2B). All of the lipid molecules that bind with PICK1 are negatively charged, in agreement with the idea that positively charged residues of the BAR domain interact with negatively charged lipids. crescent-shaped dimer, and each monomer uses two pairs of positively charged residues to bind negatively charged membrane (Peter et al., 2004). One pair is located at the end of the crescent dimer, whereas the other pair is located at the concave surface of the dimer. Based on sequence alignments, lysines 266 and 268 of PICK1 are likely to be the pair of positively charged residues located at the end of the crescent dimer and responsible for lipid binding (supplemental Fig. S1 B, available at www.jneurosci.org as supplemental material). We changed both lysines 266 and 268 to negatively charged glutamate and tested the lipid binding capability of the mutant (PICK1 2K-E). As shown in Figure $1 C$,
The negative and positive regulation of the lipid binding capability of PICK1

The finding that the $5 \mathrm{~K}-\mathrm{E}$ mutation completely eliminated the lipid binding capability of PICK1 indicates that the BAR domain of PICK1 is necessary for its lipid binding. To test whether the BAR domain of PICK1 is sufficient for lipid binding, we generated the BAR domain only (PICK1-147-358) PICK1 fusion protein and tested its lipid binding capability. We found that the BAR domain itself is fully capable of binding to lipids with slightly higher lipid binding capability compared with full-length PICK1 (Fig. 3). 
In view of the evidence that the BAR domain only PICK1 binds to lipid better than full-length PICK1, we suspected that other domains of PICK1 may regulate the lipid binding of its BAR domain. The $\mathrm{C}$ terminus of PICK1 contains a stretch of negatively charged acidic residues (Fig. 3). One possibility is that this negatively charged acidic domain of PICK1 could bind to the positively charged BAR domain residues and inhibit the lipid binding of the protein. To test this hypothesis, we generated a PICK1 fusion protein with its C-terminal domain deleted (PICK1-1358). We found that the binding of the PICK1-1-358 fusion protein to lipids was significantly stronger than that of fulllength PICK1, indicating that the $\mathrm{C}$ terminus of PICK1 indeed serves as an autoinhibitory domain to the lipid binding of PICK1.

To further examine the intramolecular regulation of the lipid binding capability of PICK1, we generated more deletion mutants of PICK1. We found that deleting the PDZ domain of PICK1 (PICK1-110-416) decreased its lipid binding (Fig. 3). We further deleted the 37 amino acid linker region between the PDZ domain and the BAR domain (PICK1-147-416). Deletion of this linker region almost completely diminished the lipid binding of PICK1 in the presence of the C-terminal autoinhibitory domain. It is not clear how the PDZ and the linker region enhance the PICK1 lipid binding capability. The fact that the $5 \mathrm{~K}-\mathrm{E}$ BAR domain mutant does not bind to lipids argues against the possibility that the PDZ domain or the linker region may directly bind to lipid. It is more likely that the PDZ domain and the linker region may affect the conformation of the BAR domain and consequently enhance its lipid binding capability. It has been reported that in certain BAR domains (e.g., the $\mathrm{N}$-BAR domain), a short sequence $\mathrm{N}$ terminal to these BAR domains enhances their lipid binding capabilities (Peter et al., 2004). Our result suggests that the BAR domain of PICK1 could be similar to the N-BAR domains found in other proteins.

Lipid binding is critical for subcellular localization and synaptic targeting of PICK1

We then asked what might be the function of PICK1 lipid binding. One possible function is that the lipid binding of PICK1 may regulate its subcellular localization. To test that, we transfected wild-type and lipid binding-deficient mutants of PICK1 into HEK293T cells and examined their subcellular localization. GFPtagged wild-type PICK1, when transfected into HEK293T cells, was mainly diffuse in the cytosol, with a small number of clusters in some cells (supplemental Fig. S2 A, available at www.jneurosci.org as supplemental material). These clusters were usually

B
A wt PICK1

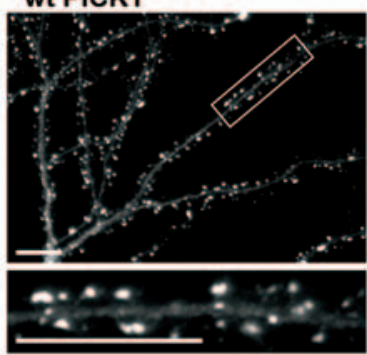

PICK1 5K-E
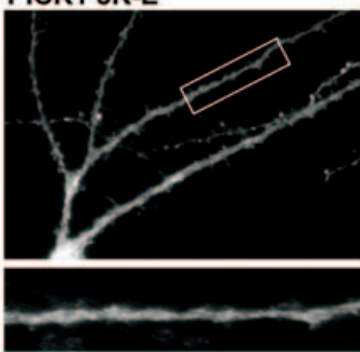

PICK1 2K-E

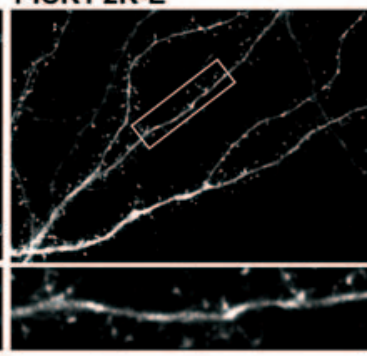

PICK1 KD-AA

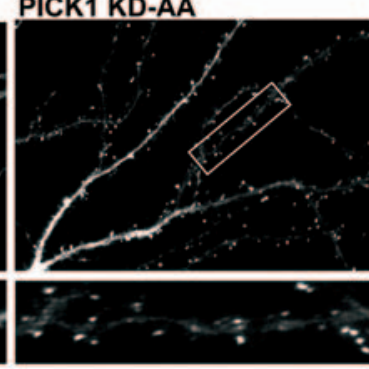

PICK1 3K-E

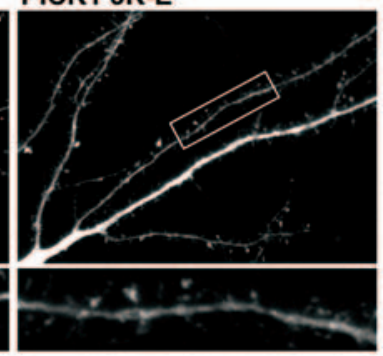

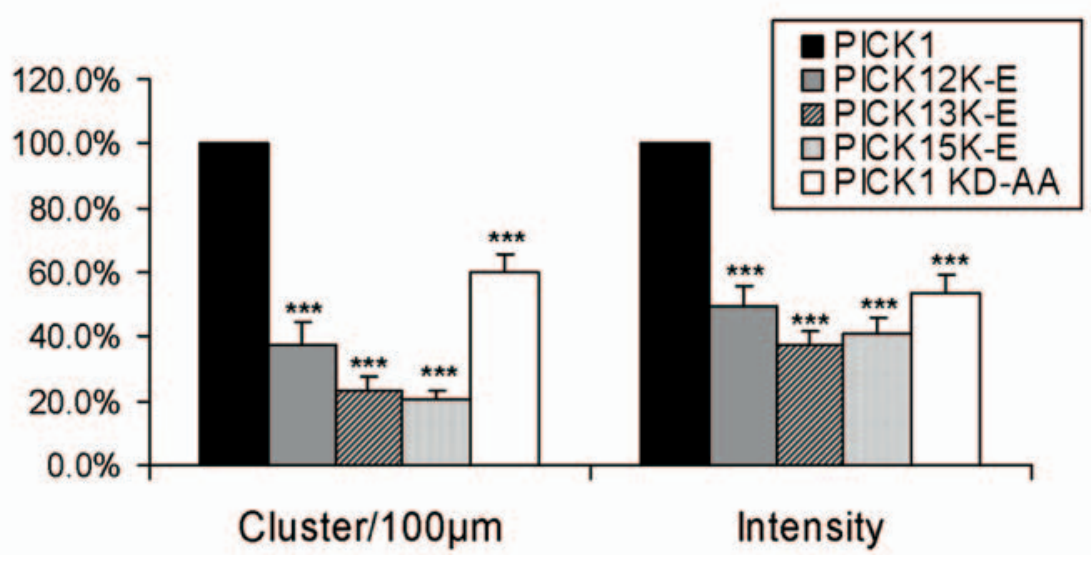

Figure 4. Lipid binding is important for synaptic targeting of PICK1.A, GFP-PICK1 and different mutants were introduced into neurons using the Sindbis viral expression system. One day after infection, neurons were fixed and visualized with a fluorescence microscope. Wild-type GFP-PICK1 was highly clustered along the dendrites. In contrast, the lipid binding-deficient mutants of PICK1 (2K-E, 3K-E, and 5K-E) had dramatically reduced clusters. The PDZ domain mutant PICK1 (KD-AA) still had significant a mounts of clusters, albeit less than wild-type PICK1. Scale bar, $10 \mu \mathrm{m}$. B, Numbers and intensities of PICK1 clusters were clusters compared with wild-type PICK1 (*** $<0.001 ; t$ test). Although the PDZ mutant of PICK1 (KD-AA) also had considerably fewer clusters than wild-type PICK1 $(p<0.001)$, it still had significantly more clusters compared with all the lipid bindingdeficient mutants $(p<0.001)$. Error bars represent SEM.

distributed between the nuclear membrane and the plasma membrane. In contrast, when we transfected lipid binding-deficient mutants into HEK293T cells, these clusters essentially disappeared (supplemental Fig. S2 A, available at www.jneurosci.org as supplemental material). We quantified the result and found that the percentages of cells that had at least one cluster were significantly lower for lipid binding-deficient mutants of PICK1 (wildtype PICK1, $40.0 \pm 0.3 \%$; $2 \mathrm{~K}-\mathrm{E}, 9.2 \pm 1.6 \%$; $3 \mathrm{~K}-\mathrm{E}, 8.7 \pm 1.7 \%$; $5 \mathrm{~K}-\mathrm{E}, 9.4 \pm 1.2 \%$; $p<0.01$ ) (supplemental Fig. S2 B, available at www.jneurosci.org as supplemental material). Similar distribution patterns were also observed when we transfected HEK293T cells with myc-tagged wild-type PICK1 and lipid bindingdeficient mutants (data not shown).

In neurons, PICK1 has been shown to be highly enriched at 
A
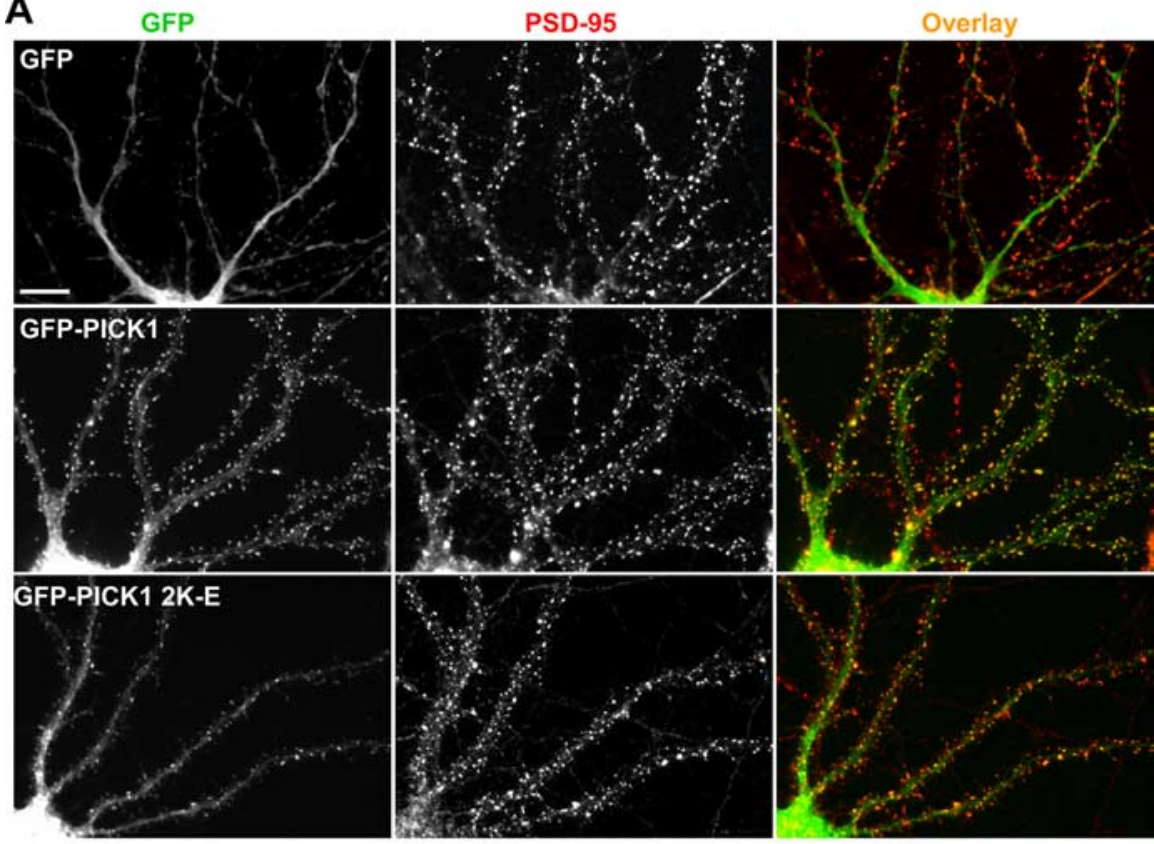

B

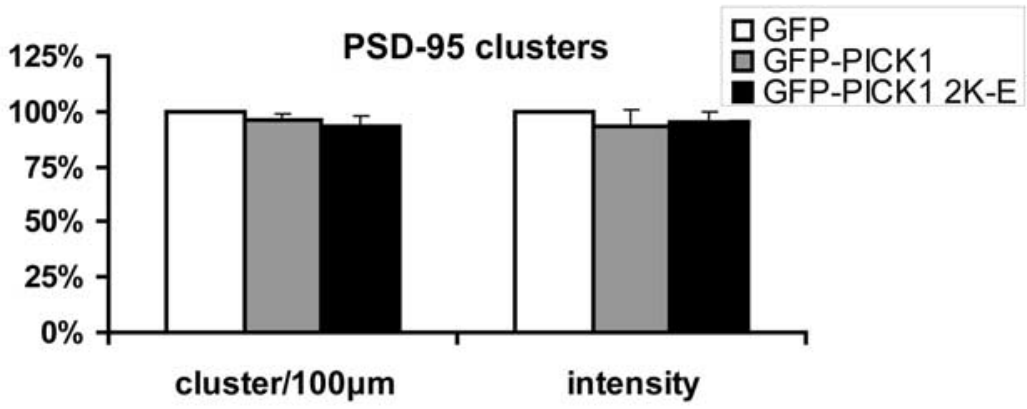

Figure 5. PICK1 colocalizes but does not affect the number or intensity of PSD-95 clusters at the synapses. $\boldsymbol{A}$, GFP alone, GFP-PICK1, and GFP-PICK1 2K-E were infected to hippocampal neurons and stained with antibody to PSD-95. Wild-type PICK1 is highly clustered and colocalizes with PSD-95 very well. PICK1 2K-E is mainly missing from PSD-95 clusters. Scale bar, $10 \mu \mathrm{m}$. $\boldsymbol{B}$ Quantification results indicate that neither wild-type PICK1 nor PICK1 2K-E significantly change the number or intensity of PSD-95 clusters. Error bars represent SEM.

excitatory synapses (Xia et al., 1999). We wondered whether the lipid binding of PICK1 affects its synaptic targeting. We examined the synaptic localization of lipid binding-deficient mutants of PICK1 compared with wild-type PICK1. GFP-tagged wildtype and lipid binding-deficient mutants of PICK1 were introduced into cultured hippocampal neurons using the Sindbis viral expression system. As shown in Figure 4A, wild-type GFP-PICK1 was highly clustered along the dendrites, mimicking the synaptic localization of endogenous PICK1, and colocalized with PSD-95 at synapses (Fig. 5A). In contrast, the lipid binding-deficient mutants were found to form far fewer clusters in infected neurons (Fig. 4A). We quantified both the number and intensity of PICK1 clusters. The number of clusters of lipid binding-deficient mutants was significantly reduced compared with that of wild-type PICK1 (the number of clusters per $100 \mu \mathrm{m}$ of dendrite was normalized against wild-type PICK1, which was set at 100\%; $2 \mathrm{~K}-\mathrm{E}$, $37.2 \pm 7.5 \%$; $3 \mathrm{~K}-\mathrm{E}, 23.6 \pm 3.9 \%$; $5 \mathrm{~K}-\mathrm{E}, 20.1 \pm 3.3 \%$; $p<0.001$ ) (Fig. $4 \mathrm{~B}$ ). The cluster intensities of the $2 \mathrm{~K}-\mathrm{E}, 3 \mathrm{~K}-\mathrm{E}$, and $5 \mathrm{~K}-\mathrm{E}$ mutants were also significantly reduced (the cluster intensity of wild-type PICK1 was normalized to $100 \%$; $2 \mathrm{~K}-\mathrm{E}, 49.7 \pm 6.0 \%$;
$3 \mathrm{~K}-\mathrm{E}, 37.7 \pm 3.7 \%$; $5 \mathrm{~K}-\mathrm{E}, 40.7 \pm 5.4 \%$; $p<0.001)$. We also compared the synaptic targeting of the lipid binding-deficient mutants with PICK1 PDZ mutant (PICK1 KD-AA), which has been shown previously to have reduced synaptic targeting (Boudin and Craig, 2001). We found that although the PICK1 PDZ mutant also has a reduced number and reduced intensity of synaptic clusters, its synaptic targeting is significantly better than that of the lipid binding-deficient mutants $(60.3 \pm 5.2$ and $53.4 \pm 5.9 \%$ of wild-type PICK1 for cluster number and intensity, respectively; $p<$ 0.001). This result indicates that the lipid binding of PICK1 could be a more fundamental determinant of the synaptic targeting of PICK1. It is interesting to note that deletion of the acidic domain of PICK1 has been reported to enhance its synaptic targeting (Boudin and Craig, 2001). In view of our result that deletion of the acidic domain enhances the lipid binding capability of PICK1, we believe this enhancement of PICK1 synaptic targeting is likely to be the consequence of its increased lipid binding capability. These results indicate that synaptic targeting of PICK1 correlates well with its lipid binding capability.

To rule out the possibility that the change in synaptic PICK1 is a consequence of changing the number of spines or postsynaptic structures, we stained PSD95, a well-characterized postsynaptic marker, together with wild-type PICK1 and the PICK1 2K-E mutant. We found that wild-type PICK1 colocalized very well with PSD-95 clusters, whereas PICK1 $2 \mathrm{~K}-\mathrm{E}$ was essentially missing from these clusters (Fig. 5A). Quantification results show that neither wild-type PICK1 nor PICK1 2K-E mutant significantly changed the number or intensity of PSD-95 clusters (with GFP-infected neurons normalized to $100 \%$, the cluster number per $100 \mu \mathrm{m}$ of wild-type PICK1, $95.7 \pm 3.5 \%$, 2K-E, $93.5 \pm 4.6 \%$; the cluster intensity of wild-type PICK1, $93.5 \pm$ 7.1\%, 2K-E, $95.6 \pm 4.2 \%$; $n=37$ ) (Fig. 5B).

\section{Lipid binding of PICK1 is important for clustering and synaptic targeting of AMPA receptors}

We then investigated the function of PICK1 lipid binding in AMPA receptor trafficking. PICK1 has been shown to cluster AMPA receptors when cotransfected into HEK293T cells (Dev et al., 1999; Xia et al., 1999). We wondered whether the lipid binding capability of PICK1 might play a role in this process. As shown in supplemental Figure S3A (available at www.jneurosci. org as supplemental material), when wild-type PICK1 was cotransfected into HEK293T cells together with AMPA receptor subunit GluR2, PICK1 and GluR2 formed many coclusters in discrete sites of the cytosol. The localization of these clusters is similar to that of the PICK1 self-clusters but with greatly increased number and size. The number of these clusters can range from a few to $>100$ in a single cell. In contrast, when PICK1 2K-E 
was cotransfected with GluR2, we did not observe any coclusters (supplemental Fig. $\mathrm{S} 3 B$, available at www.jneurosci.org as supplemental material). Similarly, no cocluster with GluR2 was observed for PICK1 3K-E and 5K-E mutants. The quantification result showed that $78.1 \pm 4.5 \%$ of cells cotransfected with GluR2 and wildtype PICK1 had coclusters, whereas the lipid binding-deficient mutants had no coclusters at all. To rule out the possibility that the loss of GluR2 clustering with PICK1 2K-E was the consequence of lost interaction of PICK1 with GluR2 or loss of PICK1 self-association capability, we performed yeast two-hybrid experiments and found that PICK1 2K-E can still interact with GluR2 and self-associates (supplemental Fig. S4, available at www.jneurosci. org as supplemental material). We also tested the interaction of PICK1 2K-E with GRIP/ABP, another AMPA receptor associating protein, because a recent report showed that PICK1 BAR domain binds to GRIP/ABP (Lu and Ziff, 2005). We found that PICK1 2K-E interacts with GRIP/ABP in a way similar to wild-type PICK1 (supplemental Fig. S5, available at www. jneurosci.org as supplemental material). These results indicate that the lipid binding capability is required for PICK1-induced clustering of AMPA receptors.

In neurons, PICK1 colocalizes with AMPA receptors at excitatory synapses (Xia et al., 1999). Our findings that the lipid binding of PICK1 is required for its synaptic targeting and its clustering of AMPA receptors suggests that lipid binding of PICK1 may also regulate targeting of AMPA receptors to synapses. To test this possibility, we introduced wild-type and lipid binding-deficient mutant PICK1 $2 \mathrm{~K}-\mathrm{E}$ into cultured neurons. We found that wild-type PICK1 can increase the synaptic targeting of GluR2 (Fig. 6A). Quantification results showed that PICK1 significantly increased the number and intensity of GluR2 clusters to $125.5 \pm 6.7$ and $118.3 \pm 4.7 \%$, respectively, compared with GFP-infected neurons, which were normalized to $100 \%(n=70 ; p<0.0)$ (Fig. 6C). In contrast, the synaptic clusters of GluR2 in neurons infected with lipid bindingdeficient mutant PICK1 $2 \mathrm{~K}$-E were similar to those of GFPinfected neurons but were significantly fewer and weaker compared with those of neurons infected with wild-type PICK1 (cluster/100 $\mu \mathrm{m}, 99.5 \pm 6.1 \%$; intensity, $103.1 \pm 4.2 \% ; n=70$; $p<0.05$ compared with wild-type PICK1) (Fig. 6C).

We also examined the effect of wild-type PICK1 and lipid binding-deficient mutants of PICK1 in synaptic targeting of GluR1, which can form complexes with GluR2. We found that, similarly to GluR2, wild-type PICK1 also significantly increased the number and intensity of synaptic GluR1, whereas the lipid binding-deficient PICK1 2K-E mutant did not (Fig. 6B,D) (the value from GFP-infected neurons was normalized to $100 \%$;
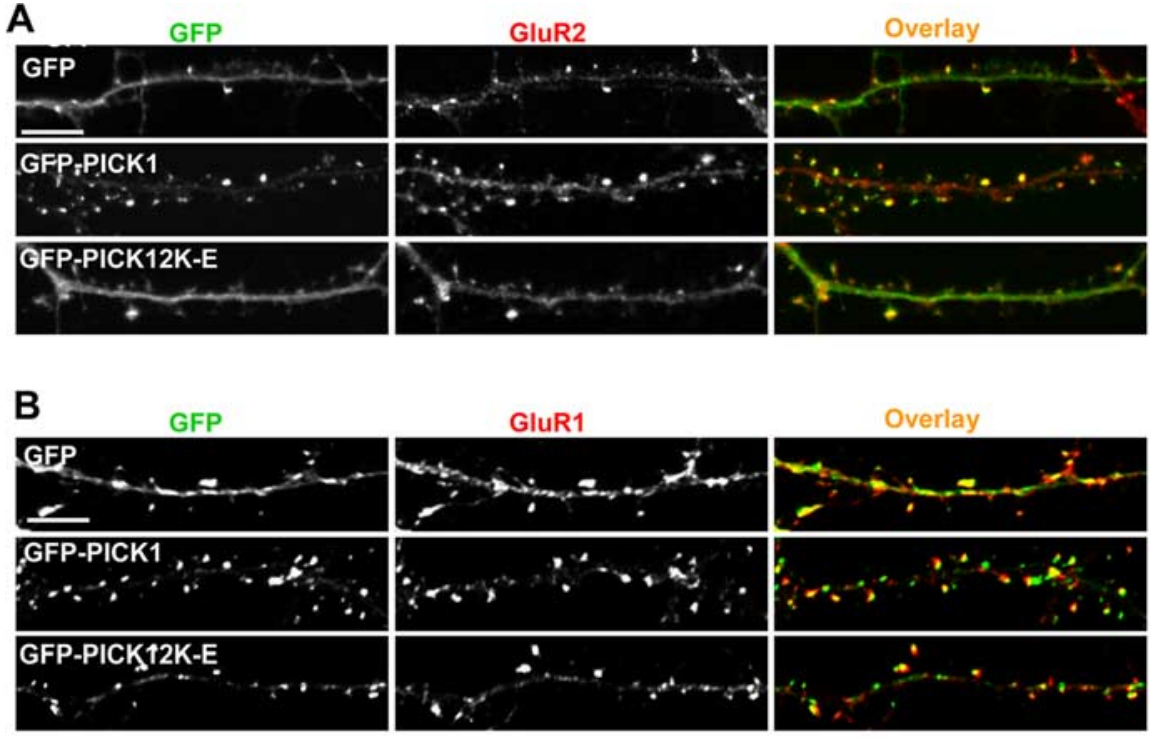

Figure 6. Lipid binding of PICK1 regulates synaptic targeting of GluR2 and GluR1. $A$, GFP, GFP-PICK1, and GFP-PICK1 2K-E were introduced into neurons using the Sindbis viral expression system. Neurons were fixed and stained with antibody against GluR2. The GluR2 clusters colocalize with the PICK1 clusters at the synapses. Neurons infected with wild-type PICK1 had substantially

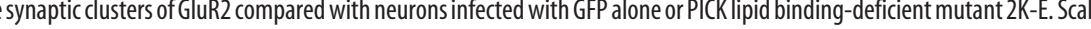

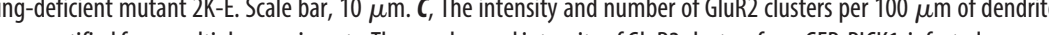
overexpression significantly increased the GluR1 cluster number $\left({ }^{* * *} p<0.001\right)$, but the lipid binding-deficient mutant 2K-E did not $(p>0.05)$. Error bars represent SEM.

GluR1 clusters number per $100 \mu \mathrm{m}$, GFP-PICK1, $188.4 \pm 12.9 \%$, GFP-PICK1 2K-E, $119.7 \pm 9.9 \%$; GluR1 cluster intensity, GFPPICK1, $153.3 \pm 6.2 \%$, GFP-PICK1 2K-E, $97.3 \pm 4.8 \% ; n=30)$. These results indicate that PICK1 has the capability to target AMPA receptors to synapses, and this capability depends on the lipid binding of PICK1.

\section{Lipid binding of PICK1 regulates the surface expression of AMPA receptors}

PICK1 has been found to regulate the surface expression of a number of proteins. We tested whether the lipid binding of PICK1 was involved in this process. This was done by transfecting wild-type PICK1 or PICK1 2K-E with GluR2 into HEK293T cells. Surface GluR2 was labeled with membrane-impermeable biotin. After lysing the cells, surface GluR2 was separated from intracellular GluR2 via binding of biotin to immobilized neutravidin. Although wild-type PICK1 substantially reduced the amount of 
A

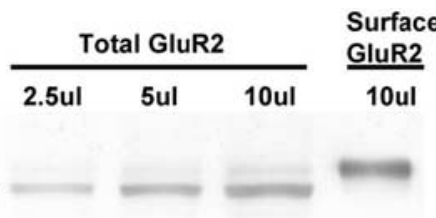

GluR2+ vector
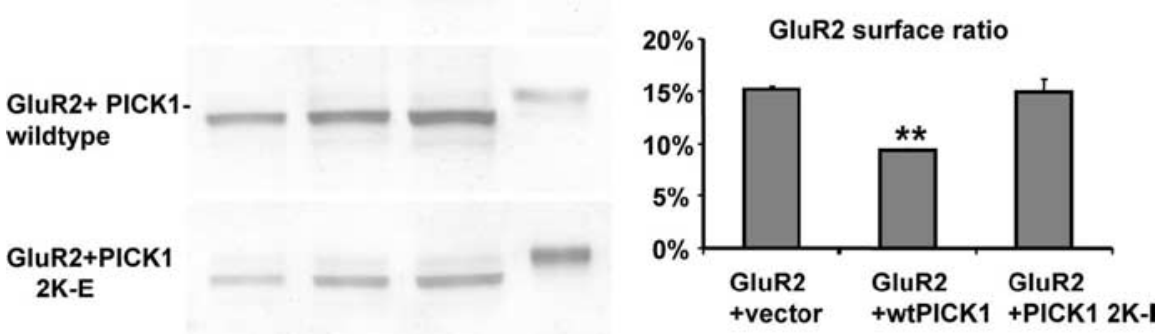

C
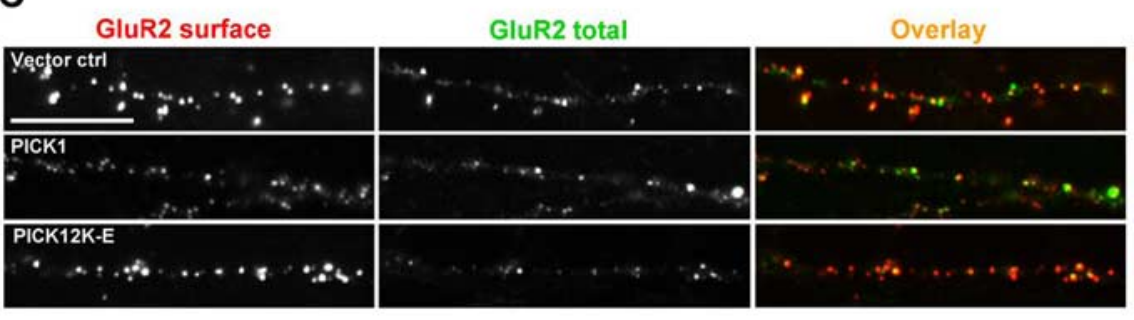

D

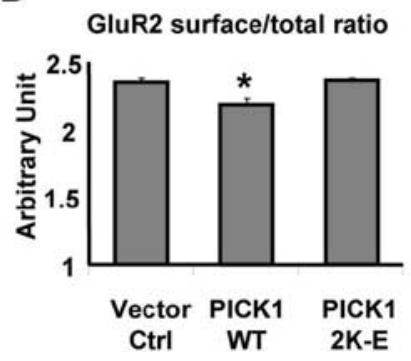

E

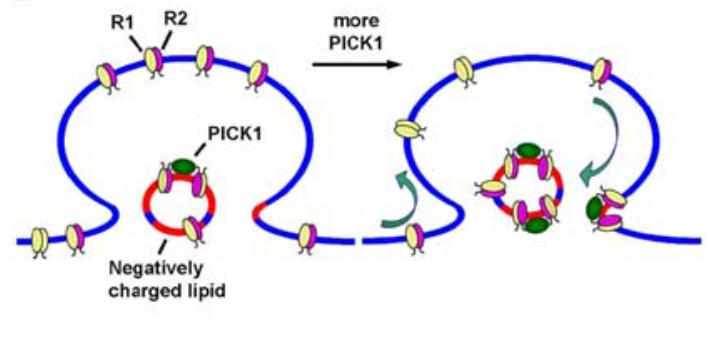

GluR2 plus PICK1-wild type, $9.4 \pm 0.1 \%$; $n=6 ; p<0.01$ ) (Fig. 7B). In contrast, PICK1 2K-E mutant did not significantly alter the surface expression of GluR2 (PICK1-2K-E, $14.9 \pm 1.3 \% ; n=6 ; p>$ $0.05)$. These results indicate that lipid binding is important for PICK1-regulated surface expression of AMPA receptors.

To further investigate the role of PICK1 lipid binding in surface expression of AMPA receptors at synapses, we performed surface staining of GluR2 and measured the surface-to-total ratio of GluR2 at the synapses. GFP-tagged GluR2 was transfected into cortical neurons together with myc-tagged wild-type PICK1 or PICK1 2K-E mutant. Surface GluR2 was labeled with GFP antibody, which was added to the neurons before fixation. After fixation, signal from the GFP antibody was visualized as surface GluR2, whereas the native GFP signal was taken as total GluR2 (Fig. 7C). The intensities of both surface and total GluR2 clusters were measured and normalized to respective dendritic gray values to obtain the relative intensities. The surface-to-total ratio of GluR2 clusters was determined by dividing the relative surface intensity by the total intensity of individual clusters. We found that wild-type PICK1 significantly decreased the ratio of GluR2 on the synaptic surface, whereas PICK1 2K-E mutant did not (surface:total ratio: vector control, $2.36 \pm$ 0.02; wild-type PICK1, $2.19 \pm 0.04$; PICK1 $2 \mathrm{~K}-\mathrm{E}, 2.36 \pm 0.02 ; n=30$ ) (Fig. 7D). The findings that PICK1 increases the total (Fig. 6) but reduces the surface ratio of AMPA receptors at synapses suggest that PICK1 maintains an intracellular reserve pool of AMPA receptors at synapses in a lipid binding-dependent manner.

\section{Long-term depression in hippocampal neurons requires lipid binding of PICK1}

PICK1 has been shown to be important for LTD (Xia et al., 2000; Kim et al., 2001). To investigate whether lipid binding of PICK1 plays any role in LTD, we intracellularly perfused wild-type PICK1 and lipid binding-deficient mutant PICK1 2K-E fusion proteins into hippocampal CA1 pyramidal neurons using whole-cell patch pipettes. We noticed that whereas the control protein or wild-type PICK1 did not change the baseline, PICK1 2K-E fusion proteins caused a significant increase

surface GluR2 (Fig. 7A, comparison of surface GluR2 in the top and middle rows), PICK1 2K-E did not (Fig. 7A, bottom row). The experiment was repeated, and the data were quantified by densitometry. We found that wild-type PICK1 significantly reduced the surface ratio of GluR2 (GluR2 alone, $14.6 \pm 0.4 \%$; in the basal excitatory postsynaptic current (EPSC) during the first $10 \mathrm{~min}$ of recording (control, $100.2 \pm 4.6 \%, n=20$; wildtype PICK, $102.0 \pm 4.4 \%, n=25$; PICK1 2K-E, $144.7 \pm 6.7 \%$, $n=29$ ) (Fig. $8 A, B$ ). This is likely because of a release of AMPA 


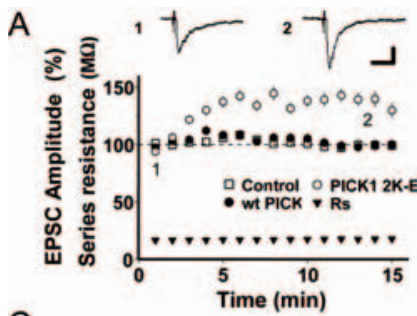

C
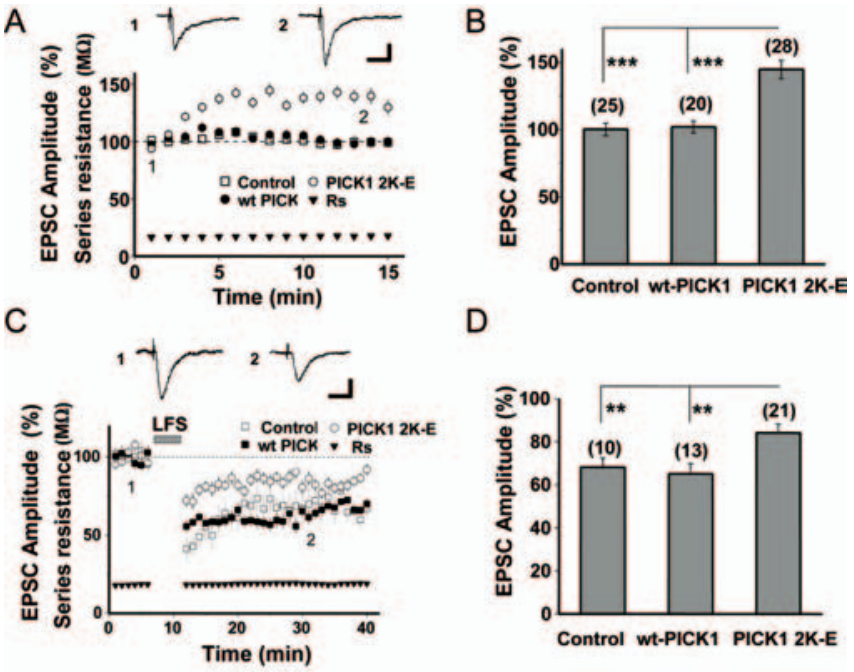

D

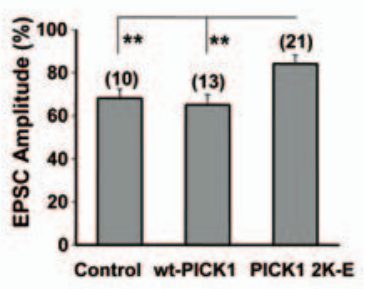

Figure 8. Lipid binding of PICK1 is important for long-term depression. A, Application of the PICK1 2K-E mutant (open circles) through patch pipettes increased the EPSC amplitude that reached a steady-state level within $10 \mathrm{~min}$. Data were collected immediately after the establishment of whole-cell recording and normalized in each neuron by the mean amplitude of the EPSCs observed during the initial 2 min of recording. $B$, Summary of the results from the experiments shown in $A$. Data represent averaged EPSCs recorded during the period from 7 to $15 \mathrm{~min}$ after the establishment of whole-cell recording and are normalized as in $\boldsymbol{A}$. The number associated with each column refers to the number of neurons tested in each condition. C, Inhibition of low-frequency stimulation (LFS)-induced LTD by the PICK1 2K-E mutant. EPSCs were recorded $10 \mathrm{~min}$ after the establishment of whole-cell recording and normalized by the mean amplitude of EPSCs recorded during the control period (before LFS application). D, Summary of the results from experiments shown in $C$. Data represent averaged EPSCs recorded 20 min after LFS application and are normalized as in $C$. The number associated with each column refers to the number of neurons tested in each condition. $\boldsymbol{A}, \boldsymbol{C}$, Insets, Sampled traces of averaged EPSCS in the PICK1 2K-E group $(\boldsymbol{A})$ and the control group $(\boldsymbol{C})$, respectively, recorded during the period indicated by the corresponding numbers. Calibration: $A, 50 \mathrm{pA}, 20 \mathrm{~ms}$; $C, 50 \mathrm{pA}, 30 \mathrm{~ms} .{ }^{* *} \mathrm{p}<$ $0.01 ;{ }^{* * *} p<0.001$. Error bars represent SEM.

receptors from the reserve pool. Because PICK1 2K-E cannot bind to lipid, it will displace the AMPA receptors from the reserve pool and lead to their insertion to synaptic surface.

After the EPSCs were stabilized, we then induced LTD using the pairing protocol (300 stimuli; $1 \mathrm{~Hz}$ at $-40 \mathrm{mV}$ ) (Dudek and Bear, 1992; Mulkey and Malenka, 1992; Daw et al., 2000). In neurons perfused with control fusion protein or wild-type PICK1, we can reliably obtain LTD (normalized EPSC amplitude: control, $68.1 \pm 4.3 \%, n=10$; wild-type PICK1, $65.1 \pm 4.7 \%, n=$ 13) (Fig. 8C,D). In contrast, in neurons perfused with PICK1 2K-E mutant, LTD is significantly less compared with either the control or wild-type PICK1 perfused neurons (PICK1 2K-E, $84.1 \pm 4.1 \% ; n=21 ; p<0.01)$. These data indicate that lipid binding of PICK1 contributes to the expression of LTD, likely by regulating AMPA receptor trafficking during synaptic plasticity.

\section{Discussion}

In this study, we demonstrated that PICK1 can directly bind to lipids via its BAR domain. The lipid molecules that bind to PICK1 are mainly negatively charged phosphoinositides. Mutations of critical residues within the PICK1 BAR domain disrupt its lipid binding. Moreover, lipid binding of the BAR domain is negatively regulated by the acidic domain and positively regulated by the PDZ domain and the linker region of PICK1. We also provide evidence for two potential physiological functions of PICK1 lipid binding. The first is that lipid binding is critical to the subcellular localization of PICK1 in cells. In HEK293T cells, lipid bindingdeficient mutants of PICK1 do not concentrate in discrete cyto- solic sites as wild-type PICK1 does. As a consequence, these mutants cannot cluster the PICK1 PDZ binding partner GluR2 in HEK293T cells. In neurons, the lipid binding-deficient mutants do not target to synapses as wild-type PICK1 does. We found that whereas PICK1 can increase synaptic targeting of AMPA receptors, lipid binding-deficient PICK1 loses the ability to target AMPA receptors to synapses. The second function is that PICK1 lipid binding is important for the surface expression of AMPA receptors. Without the lipid binding capability, PICK1 loses its ability to regulate surface expression of AMPA receptors, which leads to deficiency in long-term depression. This suggests a mechanism for PICK1-mediated AMPA receptor trafficking: although PICK1 PDZ domain binds to the C termini of AMPA receptors, its BAR domain binds to negatively charged lipids and tethers the AMPA receptors as cargo to curved-membrane microdomains that are designated to form trafficking vesicles (Fig. $7 E$ ). We believe this mechanism may not be limited to PICK1mediated AMPA receptor trafficking. Because PICK1 interacts with a number of membrane proteins, it might be a general mechanism for PICK1 to regulate the trafficking of membrane proteins via the lipid binding capability of its BAR domain.

It has been reported that overexpression of PICK1 leads to reduction of surface GluR2 at synapses (Perez et al., 2001; Terashima et al., 2004). Our result that PICK1 increases the total but reduces the surface-to-total ratio of AMPA receptors at synapses in a lipid binding-dependent manner suggests that PICK1 maintains an intracellular pool of AMPA receptors at synapses by binding to negatively charged lipids (Fig. 7E). This intracellular pool of AMPA receptors maintained by PICK1 could be the reservoir for AMPA receptor traffic to and from synaptic surface during synaptic plasticity (Daw et al., 2000; Xia et al., 2000). This notion is supported by the data that disruption of the PICK1 interaction with GluR2 or the PICK1 lipid binding capability impairs LTD, because these manipulations block the movement of AMPA receptors from the surface to the intracellular pool at synapses. The model is also in agreement with the recent finding that PICK1 knock-out mice are deficient in the activitydependent insertion of GluR2 containing AMPA receptors (Gardner et al., 2005), which could be explained by a depletion of the intracellular reserve pool of AMPA receptors in PICK1 knock-out mice. It is interesting to note that both synaptic targeting and surface expression of AMPA receptors are dependent on the lipid binding of PICK1. It is conceivable that during LTP or LTD induction, certain intracellular signal transduction pathways may change the lipid composition or affinity of PICK1 to certain lipids and thus regulate the ratio between the surface and intracellular pool of AMPA receptors at synapses. In fact, one lipid kinase, phosphatidyl inositol 3-kinase, has been reported to be involved in AMPA receptor trafficking during synaptic plasticity (Man et al., 2003).

In this study, we provided the first evidence that PICK1 increases both total GluR2 and GluR1 at synapses (Fig. 6). Although GluR1 does not directly interact with PICK1, the majority of GluR1 in neurons is coupled with GluR2 and is likely to traffic together with GluR2. However, this finding seems at odds with previous reports that PICK1 only affects surface GluR2, not GluR1 (Terashima et al., 2004; Lu and Ziff, 2005). It is even more surprising that PICK1 increases total GluR1 more than GluR2. However, this seeming discrepancy can be explained by the model that PICK1 maintains an intracellular synaptic pool of AMPA receptors, and this pool exchanges AMPA receptors with the surface synaptic pool and surface extrasynaptic pool. When overexpressing PICK1, excessive PICK1 will move GluR1 and 
GluR2 heteromeric receptors from the surface synaptic pool to the intracellular synaptic pool. Extrasynaptic AMPA receptors, especially GluR1 homomeric receptors, then move to the synaptic surface by lateral diffusion to replace the internalized receptors. This leads to an increase of total AMPA receptors, especially GluR1, at the synapses and decrease of surface GluR2 but not surface GluR1 at the synapses (Fig. 7E). This model is also in agreement with that reported by Tereshima et al. (2004) in which overexpression of PICK1 reduced surface GluR2 but increased synaptic AMPA current and inward rectification, indicating more GluR1 homomeric receptors moved to the synaptic surface. However, it should be noted that the larger effect of PICK1 on GluR1 than GluR2 should be taken with caution. We noticed that the GluR2 antibody has a high background signal on the dendrites and does not stain synapses as strongly as GluR1 antibody. The high background level of GluR2 antibody may have averaged down the signal and made the effect of PICK1 on GluR2 appear to be smaller. Additional studies are required to further clarify the role of PICK1 in GluR1 and GluR2 trafficking.

In a recent study, the BAR domain of PICK1 was found to interact with GRIP/ABP, a PDZ domain-containing protein that also interacts with GluR2/3 (Lu and Ziff, 2005). The interaction of PICK1 and GRIP/ABP was also found to be important for AMPA receptor trafficking. We found that PICK1 2K-E, the lipid binding-deficient mutant used in this study, binds to GRIP/ABP in a similar way as wild-type PICK1 (supplemental Fig. S5, available at www.jneurosci.org as supplemental material). This indicates that the effect we observed using the PICK1 2K-E mutant is likely a result of lipid binding, instead of disrupting the interaction of PICK1 with GRIP/ABP. Apparently, the lipid binding region of the PICK1 BAR domain is distinct from its binding region with GRIP. It would be interesting to determine in future studies whether there was any interplay between PICK1 interaction with lipids and its interaction with GRIP/ABP.

Many of the BAR domain-containing proteins, such as amphiphysin and endophilins, are accessory proteins in endocytosis (Marsh and McMahon, 1999; Slepnev and De Camilli, 2000; Peter et al., 2004). These accessory proteins do not directly bind to cargo proteins during endocytosis. Instead, they form complexes with the core endocytotic proteins. For example, amphiphysin was found to interact with AP2, clathrin, dynamin, and synaptojanin to regulate the endocytosis of synaptic vesicles (Wigge and McMahon, 1998; Slepnev and De Camilli, 2000). The BAR domain of amphiphysin can sense and bend the lipid bilayer to help form trafficking vesicles (Peter et al., 2004). In contrast to amphiphysin, PICK1 has a PDZ domain in addition to its lipid binding BAR domain. The PDZ domain is a well-characterized protein motif that interacts with the $C$ termini of many membrane proteins (Sheng and Sala, 2001). This suggests that PICK1, unlike other BAR domain proteins, could directly bind to cargo proteins for endocytosis.

The interaction with synaptic scaffolding proteins has been proposed as an important mechanism for targeting of synaptic proteins. For example, PSD-95, the prototypical PDZ domaincontaining protein and a major component of postsynaptic density, plays a central role in assembling the postsynaptic protein complex by interacting with a number of receptors, signaling molecules, and proteins linked to the cytoskeleton (Sheng and Sala, 2001). Protein-protein interactions have also been found to be important for synaptic targeting of PICK1. Mutations of the PDZ domain and the coiled-coil domain, both are protein-protein interaction motifs, reduce the synaptic clusters of PICK1 (Boudin and Craig, 2001). Our finding that the lipid binding of
PICK1 is required for its synaptic targeting provides a new mechanism for targeting proteins to synapses. It should be noted that lipids have been found previously to be important for targeting of synaptic proteins. PSD-95 was found to be palmitoylated and integrated into membranes after palmitoylation (Craven et al., 1999). This palmitoylation was found to be important for synaptic targeting of PSD-95 and regulation of synaptic strength (Topinka and Bredt, 1998; El-Husseini Ael et al., 2002). In contrast to the palmitoylation of PSD-95, which covalently links the protein with fatty acid, the interaction of the PICK1 BAR domain with lipids is based on a noncovalent interaction, which makes the association of PICK1 with lipids more dynamic. It is conceivable that PICK1 can be recruited to a specific membrane region by adding negatively charged lipids and also easily dissociated when the membrane becomes less charged. This gives PICK1 the ability to regulate the surface expression of membrane proteins at specific times and locations, which is crucial for physiological functions such as synaptic plasticity.

\section{References}

Barry MF, Ziff EB (2002) Receptor trafficking and the plasticity of excitatory synapses. Curr Opin Neurobiol 12:279-286.

Boudin H, Craig AM (2001) Molecular determinants for PICK1 synaptic aggregation and mGluR7a receptor coclustering: role of the PDZ, coiledcoil, and acidic domains. J Biol Chem 276:30270-30276.

Bredt DS, Nicoll RA (2003) AMPA receptor trafficking at excitatory synapses. Neuron 40:361-379.

Chung HJ, Xia J, Scannevin RH, Zhang X, Huganir RL (2000) Phosphorylation of the AMPA receptor subunit GluR2 differentially regulates its interaction with PDZ domain-containing proteins. J Neurosci 20:7258-7267.

Craven SE, El-Husseini AE, Bredt DS (1999) Synaptic targeting of the postsynaptic density protein PSD-95 mediated by lipid and protein motifs. Neuron 22:497-509.

Daw MI, Chittajallu R, Bortolotto ZA, Dev KK, Duprat F, Henley JM, Collingridge GL, Isaac JT (2000) PDZ proteins interacting with C-terminal GluR2/3 are involved in a PKC-dependent regulation of AMPA receptors at hippocampal synapses. Neuron 28:873-886.

Dev KK, Nishimune A, Henley JM, Nakanishi S (1999) The protein kinase C alpha binding protein PICK1 interacts with short but not long form alternative splice variants of AMPA receptor subunits. Neuropharmacology 38:635-644.

Dudek SM, Bear MF (1992) Homosynaptic long-term depression in area CA1 of hippocampus and effects of $N$-methyl-D-aspartate receptor blockade. Proc Natl Acad Sci USA 89:4363-4367.

El-Husseini Ael D, Schnell E, Dakoji S, Sweeney N, Zhou Q, Prange O, Gauthier-Campbell C, Aguilera-Moreno A, Nicoll RA, Bredt DS (2002) Synaptic strength regulated by palmitate cycling on PSD-95. Cell 108:849-863.

Gardner SM, Takamiya K, Xia J, Suh JG, Johnson R, Yu S, Huganir RL (2005) Calcium-permeable AMPA receptor plasticity is mediated by subunitspecific interactions with PICK1 and NSF. Neuron 45:903-915.

Henley JM (2003) Proteins interactions implicated in AMPA receptor trafficking: a clear destination and an improving route map. Neurosci Res 45:243-254.

Kim CH, Chung HJ, Lee HK, Huganir RL (2001) Interaction of the AMPA receptor subunit GluR2/3 with PDZ domains regulates hippocampal long-term depression. Proc Natl Acad Sci USA 98:11725-11730.

Liao D, Zhang X, O’Brien R, Ehlers MD, Huganir RL (1999) Regulation of morphological postsynaptic silent synapses in developing hippocampal neurons. Nat Neurosci 2:37-43.

Lu W, Ziff EB (2005) PICK1 interacts with GRIP/ABP to regulate AMPA receptor trafficking. Neuron 47:407-421.

Malinow R, Malenka RC (2002) AMPA receptor trafficking and synaptic plasticity. Annu Rev Neurosci 25:103-126.

Man HY, Wang Q, Lu WY, Ju W, Ahmadian G, Liu L, D'Souza S, Wong TP, Taghibiglou C, Lu J, Becker LE, Pei L, Liu F, Wymann MP, MacDonald JF, Wang YT (2003) Activation of PI3-kinase is required for AMPA receptor insertion during LTP of mEPSCs in cultured hippocampal neurons. Neuron 38:611-624. 
Marsh M, McMahon HT (1999) The structural era of endocytosis. Science 285:215-220.

Matsuda S, Mikawa S, Hirai H (1999) Phosphorylation of serine-880 in GluR2 by protein kinase $\mathrm{C}$ prevents its $\mathrm{C}$ terminus from binding with glutamate receptor-interacting protein. J Neurochem 73:1765-1768.

Matsuda S, Launey T, Mikawa S, Hirai H (2000) Disruption of AMPA receptor GluR2 clusters following long-term depression induction in cerebellar Purkinje neurons. EMBO J 19:2765-2774.

Mulkey RM, Malenka RC (1992) Mechanisms underlying induction of homosynaptic long-term depression in area CAl of the hippocampus. Neuron 9:967-975.

Park M, Penick EC, Edwards JG, Kauer JA, Ehlers MD (2004) Recycling endosomes supply AMPA receptors for LTP. Science 305:1972-1975.

Perez JL, Khatri L, Chang C, Srivastava S, Osten P, Ziff EB (2001) PICK1 targets activated protein kinase $\mathrm{C} \alpha$ to AMPA receptor clusters in spines of hippocampal neurons and reduces surface levels of the AMPA-type glutamate receptor subunit 2. J Neurosci 21:5417-5428.

Peter BJ, Kent HM, Mills IG, Vallis Y, Butler PJ, Evans PR, McMahon HT (2004) BAR domains as sensors of membrane curvature: the amphiphysin BAR structure. Science 303:495-499.

Sheng M, Sala C (2001) PDZ domains and the organization of supramolecular complexes. Annu Rev Neurosci 24:1-29.

Slepnev VI, De Camilli P (2000) Accessory factors in clathrin-dependent synaptic vesicle endocytosis. Nat Rev Neurosci 1:161-172.
Song I, Huganir RL (2002) Regulation of AMPA receptors during synaptic plasticity. Trends Neurosci 25:578-588.

Takei K, Slepnev VI, Haucke V, De Camilli P (1999) Functional partnership between amphiphysin and dynamin in clathrin-mediated endocytosis. Nat Cell Biol 1:33-39.

Tarricone C, Xiao B, Justin N, Walker PA, Rittinger K, Gamblin SJ, Smerdon SJ (2001) The structural basis of Arfaptin-mediated cross-talk between Rac and Arf signalling pathways. Nature 411:215-219.

Terashima A, Cotton L, Dev KK, Meyer G, Zaman S, Duprat F, Henley JM, Collingridge GL, Isaac JT (2004) Regulation of synaptic strength and AMPA receptor subunit composition by PICK1. J Neurosci 24:5381-5390.

Topinka JR, Bredt DS (1998) N-terminal palmitoylation of PSD-95 regulates association with cell membranes and interaction with $\mathrm{K}^{+}$channel Kv1.4. Neuron 20:125-134.

Wigge P, McMahon HT (1998) The amphiphysin family of proteins and their role in endocytosis at the synapse. Trends Neurosci 21:339-344.

Xia J, Zhang X, Staudinger J, Huganir RL (1999) Clustering of AMPA receptors by the synaptic PDZ domain-containing protein PICK1. Neuron 22:179-187.

Xia J, Chung HJ, Wihler C, Huganir RL, Linden DJ (2000) Cerebellar longterm depression requires PKC-regulated interactions between GluR2/3 and PDZ domain-containing proteins. Neuron 28:499-510.

Yang Y, Ge W, Chen Y, Zhang Z, Shen W, Wu C, Poo M, Duan S (2003) Contribution of astrocytes to hippocampal long-term potentiation through release of D-serine. Proc Natl Acad Sci USA 100:15194-15199. 